\title{
Floristic Diversity of Kandi Region of Hoshiarpur, Punjab, India
}

\author{
Laxmi Rawat ${ }^{1}$, R. K. Manhas ${ }^{2, *}$, Deepak Kholiya ${ }^{1}$, S. K. Kamboj ${ }^{1}$ \\ ${ }^{1}$ Forest Ecology \& Environment Division, Forest Research Institute, Dehradun, Uttarakhand, India \\ ${ }^{2}$ Department of Botany, Govt. Degree College, Kathua, India \\ *Corresponding author: manhasrk@rediffmail.com
}

Received January 12, 2013; Revised March 24, 2013; Accepted June 07, 2013

\begin{abstract}
Present study was conducted in the Kandi region of Hoshiarpur, India. Kandi region is the transitional zone between the Siwaliks and the plains. Field trips were undertaken weekly in all parts of the study site and plants were collected during each trip. Herbaceous flora was excavated as a whole whereas in case of shrubs and trees, only the tender twigs bearing flowers and/or fruits were taken. The dried specimens were mounted on the herbarium sheets. These herbarium sheets were protected against damages by poisoning them with $1 \%$ mercuric chloride and naphthalene balls. Total 176 plant species belonging to 57 families and 133 genera were recorded from the study site. Out of these 176 plants, 175 were angiosperms and 1 was gymnosperm. The contribution of dicotyledons was $78.3 \%$ (137 species and 105 genera) and monocotyledons 21.7\% (38 species and 27 genera). Poaceae was the most dominant family with 30 species and 21 genera. Other important families were Papilionaceae, Caesalpiniaceae, Euphorbeaceae, Apocynaceae, Acanthaceae and Mimosaceae. The most dominant life form was trees (36.9\%), followed by shrubs (22.7\%), grasses (17.1\%), herbs (13.6\%), climbers (8.5\%) and sedges (1.1\%). Studies of forest flora provide useful information on several aspects related to species diversity like dominant families, life-form status etc. The researchers and forest managers can exploit this information in planning of sustainable utilization of these resources. Time to time assessment of species diversity also helps in studying the impact of temporal changes like climate change on species distribution.
\end{abstract}

\section{Keywords: grazing, life-form, papilionaceae, poaceae, shiwaliks}

Cite This Article: Rawat, Laxmi, R. K. Manhas, Deepak Kholiya, and S. K. Kamboj "Floristic Diversity of Kandi Region of Hoshiarpur, Punjab, India.” Applied Ecology and Environmental Sciences 1, no. 4 (2013): $49-54$. doi: 10.12691/aees-1-4-3.

\section{Introduction}

The Kandi region spanning from Kashmir region, Punjab and Haryana is the transitional zone between the Siwaliks and the plains [1]. Kandi area of Punjab is a submountainous zone that stretches in a thin belt along the northeastern border of the state of Punjab, and comprises the Punjab Shiwaliks and strip of undulating land below the hills in the districts of Gurdaspur, Hoshiarpur, Fatehgarh Sahib and Ropar, with a length and width of $161 \mathrm{~km}$ and $10 \mathrm{~km}$, respectively.

Punjab has $84 \%$ of its total geographical area under agriculture and only $6.07 \%$ under forests. The majority of forest area of the state is in the form of block forests in Shiwalik hills or Kandi region. Champion and Seth [2] categorize these forests as Dry Deciduous thorn scrub forests. About 83 percent of the forest area in the Kandi belongs to the local communities and private individuals. Private forests include those owned by individuals, groups of individuals or the Panchayat. The Forest Department exercises control over these forest areas under the Land Preservation Act, 1900. This area constitutes about 52 percent of the total forest areas in the State.
The pressures on the forests for fuel-wood and fodder did not allow regeneration. Fires, which generally broke out in the summer destroys whatever remains. Most of the forest areas are infested by Lantana camara and Parthenium hysterophorus, two of most obnoxious weeds of the world. For all these reasons, the Kandi region is one of the most degraded areas of Punjab. With increase in population pressure (human and cattle) and subsequent overexploitation of renewable resources has ultimately affected the carrying capacity of the system.

The earliest record of plants from Punjab are found in Stewart [3] who published a book on 'Punjab Plants' and Atchins on [4] published a list of plants of Hoshiarpur district. Other significant contributors towards the listing of flora of Punjab are Bamber [5], Parker [6], Nair [7], Sharma and Khosla [8], Sharma [9], Sharma et al. [10] and Manhas et al. [11]. Studies of forest flora provide useful information on several aspects related to species diversity, besides other valuable information like dominant families, life-form status etc. Keeping the importance of the subject in mind, the present study was undertaken in the Kandi area of Punjab with an objective of listing the plants of Kandi region of Hoshiarpur, so that the importance of the flora of this region is recognized and 
some strategies are formulated for their conservation and sustainable use.

\section{Material and Methods}

\subsection{Study Site}

Present study site is located between $31^{\circ} 31^{\prime}$ and $31^{\circ} 53^{\prime}$ $\mathrm{N}$ latitude, $75^{\circ} 55^{\prime}$ and $75^{\circ} 92^{\prime} \mathrm{E}$ longitude. It has an average elevation of $296 \mathrm{~m}$ (971 feet). May and June are the hottest months of the year during which the temperature rises to $45^{\circ} \mathrm{C}$. The average annual rainfall for the last five years is $832.32 \mathrm{~mm}$. Most of the rainfall is received during monsoon from July to September (Figure 1).

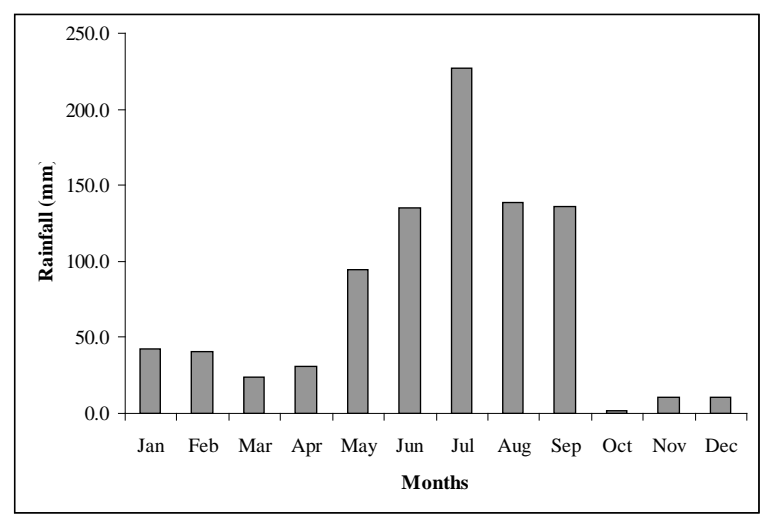

Figure 1. Rainfall (mm) of the study area for the last five years (20012005)

Hoshiarpur falls into two nearly equal portions of hill and plain country. Its eastern face consists of the westward slope of the Solar Singhi Hills; parallel with that ridge, a line of lower heights belonging to the Shiwalik Range traverses the district from south to north, while between the two chains stretches a valley of uneven width, known as the Jaswan Dun. Its upper portion is crossed by the Sohan torrent, while the Sutlej sweeps into its lower end through a break in the hills, and flows in a southerly direction until it turns the flank of the central range, and debouches westwards upon the plains.

\subsection{Methodology}

Floristic trips were undertaken weekly in all parts of the study site. Plants were collected during each trip and while collecting plants, voucher numbers was allotted to each specimen and details entered into the field notebook. Field data collected for every specimen included place of collection, altitude, date of collection, voucher number, flower color, fragrance and other such characters, which are not retained on pressing. Herbaceous flora excavated as a whole whereas in case of shrubs and trees, only the tender twigs bearing flowers and/or fruits were taken. Care was taken to collect a disease free specimen. The plants collected were pressed at the earliest in old newspapers and blotters. The pressing of plant specimens was carried out with the help of wooden press having four nut and bolts for tightening. The dried specimens were mounted on the herbarium sheets using glue and finally by stitching them at required places with the help of cotton thread. Glued pieces of paper were fixed over the knots at the back of the sheet to prevent them from getting loose with the passage of time. Herbarium sheets were protected against damage from insects by poisoning them with $1 \%$ mercuric chloride. Naphthalene balls were also used to keep the insects away from the Herbarium.

The specimens were identified either directly with the help of the herbariums of B. S. I. Northern Circle Dehradun and Herbarium of F. R. I., Dehradun or indirectly using local floras $[6,7,8,9,10]$. The voucher specimens were submitted to the Forest Department of Punjab, Chandigarh, India.

\section{Results and Discussion}

Total 176 plant species belonging to 57 families and 133 genera were recorded from the study site (Table 1). Out of 176 plants, 175 were angiosperms and 1 gymnosperm. The contribution of dicotyledons was $78.3 \%$ (137 species and 105 genera) and monocotyledons 21.7\% (38 species and 27 genera).

Table 1. List of plant species present in Hoshiarpur, Punjab

\begin{tabular}{|c|c|c|c|}
\hline Totanical names of plant species & Family & Local names & Habits \\
\hline Adhatoda vasica Linn. & Acanthaceae & Besuti, Arusha & Shrub \\
\hline Barleria cristata Linn. & Acanthaceae & Kala bansa & Shrub \\
\hline Justicia gendarussa Burm. F. & Acanthaceae & Hedge plant & Shrub \\
\hline Justicia heterocarpa T. Anders. & Acanthaceae & Had-pat & Herb \\
\hline Justicia prostrata (Cl.) Gamble & Acanthaceae & Had-pat & Herb \\
\hline Peristrophe paniculata (Forssk.) Brummitt & Acanthaceae & Peristrophe & Shrub \\
\hline Achyranthus a spera Linn. & Amarantaceae & Chirchara, Latjira & Herb \\
\hline Aerva javanica Juss ex Schult. & Amarantaceae & Boi-Kalan, Dholimundi & Herb \\
\hline Aerva lanata Juss ex Schult. & Amarantaceae & Kapur-madhura & Herb \\
\hline Aerva pseudotomentosa Biatt \& Halib. & Amarantaceae & Kamheda & Tree \\
\hline Lannea coromandelica (Houtt.) Merr. & Anacardiaceae & Kehm bal & Tree \\
\hline Mangifera indica Linn. & Anacardiaceae & Amb & Shrub \\
\hline Carissa congesta Wt. & Apocynaceae & Karaunda & Shrub \\
\hline Carissa opaca Stapf. & Apocynaceae & Garna & Shrub \\
\hline Carissa spinarum Linn. & Apocynaceae & Gan & Tree \\
\hline Holarrhena antidysenterica (L.) Wall. ex DC. & Apocynaceae & Kurchi, Karchi, Karra, Kora & Climber \\
\hline Ichnocarpus frutescens Br. & Apocynaceae & Bakar bel & Shrub \\
\hline Nerium indicum Mill. & Apocynaceae & Kaner & Climber \\
\hline Vallaris solenacea Ktze. & Apocynaceae & Dudhibel & Shrub \\
\hline Calotropis procera R. Br. & Asclepiadaceae & Desi Ak & Herb \\
\hline Ageratum conyzoides Linn. & Asteraceae & Bakari abish, Gumdrya & . \\
\hline
\end{tabular}




\begin{tabular}{|c|c|c|c|}
\hline Parthenium hysterophorus Linn. & Asteraceae & Congress, Gajar grass & Herb \\
\hline Xanthium strumarium Linn. & Asteraceae & Chirchitta & Shrub \\
\hline Bidens bipinnata Linn. & Asteraceae & Kokadi & Herb \\
\hline Cordia dichotoma Forst. f. & Boraginaceae & Lasura & Tree \\
\hline Ehretia laevis Roxb. & Boraginaceae & Chamror & Shrub \\
\hline Opunitia sp. & Cactaceae & Chhitarthor & Shrub \\
\hline Bauhenia vahlii W. \& A. & Caesalpiniaceae & Taur & Climber \\
\hline Bauhenia variegata Linn. & Caesalpiniaceae & Kachnar & Tree \\
\hline Bauhinia racemosa Lam. & Caesalpiniaceae & Kali-Kachnar, Ashta, Kosundra & Tree \\
\hline Cassia fistula Linn. & Caesalpiniaceae & Amaltas & Tree \\
\hline Cassia occidentalis Linn. & Caesalpiniaceae & Chakunda/Kasaundi & Shrub \\
\hline Cassia sophera Linn. & Caesalpiniaceae & Kasunder & Shrub \\
\hline Cassia tora Linn. & Caesalpiniaceae & Ailwan, Chakunda, Panevar & Shrub \\
\hline Parkinsonia aculeate Linn. & Caesalpiniaceae & Parkinsonia & Tree \\
\hline Cannabis sativa Linn. & Cannabidaceae & Bhang, Ganja, Charas & Shrub \\
\hline Capparis sepiaria Linn. & Capparidaceae & Hins & Climber \\
\hline Crataeva nurvala Buch.-Ham. & Capparidaceae & Parvati Plant & Tree \\
\hline Crataeva unilocularis Buch.-Ham. & Capparidaceae & Barna & Tree \\
\hline Celastrus paniculata Willd. & Celastraceae & Malkangni, Sankhiran & Climber \\
\hline Elaeodendron roxburghii Wt. \& Am. & Celastraceae & Mirgu & Tree \\
\hline Anogeissus latifolia Wall. & Combretaceae & Chhal & Tree \\
\hline Terminalia alata Heyne. & Combretaceae & Chila & Tree \\
\hline Terminalia arjuna Wt. \& Am. & Combretaceae & Arjun & Tree \\
\hline Terminalia bellirica Roxb. & Combretaceae & Bahera & Tree \\
\hline Terminalia chebula Retz. & Combretaceae & Harar & Tree \\
\hline Merremia aegyptia (Linn.) Urban & Convolvulaceae & - & Creeper \\
\hline Diospyros cordifolia Roxb. & Ebenaceae & Kaindu & Tree \\
\hline Diospyros tomentosa Roxb. & Ebenaceae & Kinu & Tree \\
\hline Echinocarpus dasycarpus Benth. & Elaeocarpaceae & Gobra, Gobria & Climber \\
\hline Emblica officinalis Gtn. & Euphorbiaceae & Anula & Tree \\
\hline Euphorbia hirta Linn. & Euphorbiaceae & Lal Dudhi & Creeper \\
\hline Euphorbia royleana Boiss. & Euphorbiaceae & Thor & Shrub \\
\hline Flueggea virosa Roxb. ex Willd. & Euphorbiaceae & Girthan & Tree \\
\hline Jatropha curcas Linn. & Euphorbiaceae & Japlita, Jangli arandi & Shrub \\
\hline Mallotus philippinensis Muell. Arg. & Euphorbiaceae & Kamal & Tree \\
\hline Putranjiva roxburghii Wall. & Euphorbiaceae & Putajan & Tree \\
\hline Ricinus communis Linn. & Euphorbiaceae & Arandi & Tree \\
\hline Flacourtia indica Merr. & Flacourtiaceae & Kangoo, Paniala, Bilangra, Kanju & Tree \\
\hline Geranium wallichianum D.Don & Geraniaceae & Laljhari, Liljahri & Herb \\
\hline Colebrookia oppositifolia J. E. Smith & Lamiaceae & - & Shrub \\
\hline Ocimum basilicum Linn. & Lamiaceae & Bantulsi & Shrub \\
\hline Perilla frutescens (Linn.) Brittom & Lamiaceae & Bhangira, Jhutela & Herb \\
\hline Loranthus sp. & Loranthaceae & Banda & Shrub \\
\hline Woodfordia fruticosa Kurz. & Lythraceae & Dhawin & Shrub \\
\hline Salmalia malabarica Schott \& Endl. & Malvaceae & Semal & Tree \\
\hline Sida acuta Burm. F. & Malvaceae & Bariara & Herb \\
\hline Sida cordata Borss.-Waalkes & Malvaceae & Bhiumli & Herb \\
\hline Sida cordifolia Linn. & Malvaceae & Kharenti & Herb \\
\hline Sida ovata Forssk. & Malvaceae & Bala & Herb \\
\hline Abutilon indicum Sw. & Meliaceae & Kanghi, Comb plant. & Shrub \\
\hline Azadirachta indica Juss. & Meliaceae & Neem, Nim & Tree \\
\hline Cedrela toona Roxb. & Meliaceae & Tun & Tree \\
\hline Melia azadirach Linn. & Meliaceae & Drek & Tree \\
\hline Cissampelos pareira Linn. & Menispermaceae & - & Climber \\
\hline Tinospora malaberica Miers & Menispermaceae & Giloe & Climber \\
\hline Albizzia lebbek Benth. & Mimosaceae & Kala siris & Tree \\
\hline Mimosa himalayana Gamble & Mimosaceae & Durghari & Climber \\
\hline Acacia catechu Willd. & Mimosaceae & Khair & Tree \\
\hline Acacia leucophloea (Roxb.) Willd. & Mimosaceae & Reru & Tree \\
\hline Acacia modesta Willd. & Mimosaceae & Phalahi & Tree \\
\hline Acacia nilotica (L.) Willd. ex Del. & Mimosaceae & Kikar & Tree \\
\hline Ficus bengalensis Linn. & Moraceae & Barh, Banayan, Bargad & Tree \\
\hline Ficus glomerata Roxb. & Moraceae & Gullar & Tree \\
\hline Ficus palmate Forssk. & Moraceae & Phaguri & Tree \\
\hline Ficus religiosa Linn. & Moraceae & Pipal & Tree \\
\hline Moringa oleifera Lamk. & Moringaceae & Sohanjna & Tree \\
\hline Eucalyptus tereticomis Sm. & Myrtaceae & Safeda & Tree \\
\hline Syzygium cum ini Skeels & Myrtaceae & Jamun & Tree \\
\hline Nyctanthes arbor-tristis Linn. & Oleaceae & Kuri & Shrub \\
\hline Oxalis comiculata Linn. & Oxalidaceae & Khatti buti & Herb \\
\hline Oxalis corymbosa DC & Oxalidaceae & Khatti buti & Herb \\
\hline Abrus precatorius Linn & Papilionaceae & Rattak & Climber \\
\hline
\end{tabular}




\begin{tabular}{|c|c|c|c|}
\hline Butea топо ррета (Lamk.) Taub. & Papilionaceae & Dhak, Palas, Palah & Tree \\
\hline Dalbergia sissoo Roxb. & Papilionaceae & Shisham, Tahli & Tree \\
\hline Desmodium triflorum DC. & $\begin{array}{l}\text { Papilionaceae } \\
\end{array}$ & Janglimethi & Herb \\
\hline Indigofera cassiodes Rottl. ex DC. & Papilionaceae & Mothi & Shrub \\
\hline Millettia auriculata Baker. & $\begin{array}{l}\text { Papilionaceae } \\
\end{array}$ & Ganj & Climber \\
\hline Mucuna pruriens DC. & Papilionaceae & Grelu & Tree \\
\hline Ougeinia oojeinensis (Roxb.) Hocht. & $\begin{array}{l}\text { Papilionaceae } \\
\end{array}$ & Sanan & Tree \\
\hline Pueraria tuberosa DC. & Papilionaceae & Salohar & Climber \\
\hline Desmodium concinnum DC. & $\begin{array}{l}\text { Papilionaceae } \\
\end{array}$ & Janglimethi & Herb \\
\hline Rhamnus triquetra Wall. & Rhamnaceae & Gir githan & Shrub \\
\hline Ziziphus mauritiana Lamk. & Rhamnaceae & Ber & Tree \\
\hline Ziziphus nummularia (Burm. f.)W. A. & Rhamnaceae & Balah & Shrub \\
\hline Oroxylum indicum Vent. & Rignoniaceae & Tatpirmga & Tree \\
\hline Pyrus pashia Buch.-Ham. & Rosaceae & Kainth & Tree \\
\hline Hym enodictyon excelsum Wall. & Rubiaceae & Banthua & Tree \\
\hline Mitragyna parvifolia Korth. & Rubiaceae & Kalam & Tree \\
\hline Wendlandia heynei Sant. \& Merch. & Rubiaceae & Pansera & Shrub \\
\hline Aegle mamelos Correa ex. Roxb. & Rutaceae & Bel, Bael & Tree \\
\hline Limonia acidissima Linn. & Rutaceae & Bilan & Tree \\
\hline Murraya exotica Linn. & Rutaceae & Nargan & Shrub \\
\hline Murraya koengii (Linn.) Spreng & Rutaceae & Mitha neem, Gandhela, Barsanga & Shrub \\
\hline Salix tetrasperma Roxb. & Salicaceae & Bedmajnu & Tree \\
\hline Casearia tomentosa Roxb. & Samydaceae & Chilla & Tree \\
\hline Dodonaea viscosa (Linn.) Jacq. & Sapindaceae & Mendru & Shrub \\
\hline Euphoria dracunculoides Lam. & Sapindaceae & Chagutputi, Kangi, Richni & Tree \\
\hline Euphoria helioscopia Linn. & Sapindaceae & Hirruseeah, Mahabi, Chatriwal, & Tree \\
\hline Euphoria longana Steud. & Sapindaceae & Dudhi & Tree \\
\hline Madhuca longifolia (Koenig) Macbride & Sapotaceae & Mahwa & Tree \\
\hline Ailanthes excelsa Roxb. & Simarubaceae & Ullinium & Tree \\
\hline Helicteres isora Linn. & Sterculiaceae & Maror phali & Shrub \\
\hline Tamarix dioica Roxb. & Tamaricaceae & Jhau & Shrub \\
\hline Grewia oppositifolia Roxb. & Tiliaceae & Dhaman & Shrub \\
\hline Trium fetta rhomboidea Jacq. & Tiliaceae & Chikti & Shrub \\
\hline Holoptelea integrifolia Planch. & Ulmaceae & Kanju, Khulen Banchilla, Dhamma & Tree \\
\hline Boehmeria platyphylla D. Don & Urticaceae & Paliara & Tree \\
\hline Broussonetia papyrifera Vent. & Urticaceae & Paper mulberry & Tree \\
\hline Moras alba Linn. & Urticaceae & Tut, Mulberry, Moru & Tree \\
\hline Gmelina arborea Roxb. & Verbenaceae & Gumhar & Tree \\
\hline Grewia sapinda Roxb. & Verbenaceae & Ban kanak & Shrub \\
\hline Lantana aculenta Linn. & Verbenaceae & Janglibutti & Shrub \\
\hline Lantana camara Linn. & Verbenaceae & Panchphuli, Kuri, Chudhyal butti & Shrub \\
\hline Vitex negunda Linn. & Verbenaceae & Bana & Shrub \\
\hline Ampelocissus latifolia Planch. & Vitaceae & Gidardakh & Climber \\
\hline Tribulus terrestris Linn. & Zygophyllaceae & Gokhru & Herb \\
\hline Pinus roxburghii Roxb. & Pinaceae & Chid & Tree \\
\hline Commelina benghalensis Linn. & Commelinaceae & Kankowa & Herb \\
\hline Cyperus esculentus Linn. & Cyperaceae & Kaseru, Dila & Sedge \\
\hline Cypenus niveus Retz. & Cyperaceae & Motha & Sedge \\
\hline Dioscorea belophylla Voigt & Dioscoreaceae & Tarar, Batendu & Herb \\
\hline Dioscorea deltoidea Wall. & Dioscoreaceae & - & Climber \\
\hline Asparagus racemosus Baker & Liliaceae & Asparagus & Shrub \\
\hline Smilax parvifolia Wall. & Liliaceae & - & Climber \\
\hline Phoenix sylvestris Roxb. & Palmae & Jangli khajur & Tree \\
\hline Alloteropsis cimicina (L.) Stapf & Poaceae & Takri & Grass \\
\hline Aristida setacea Retz. & Poaceae & Ghian & Grass \\
\hline Arundinaria falcata Nees & Poaceae & Nara & Grass \\
\hline Arundo donax Linn. & Poaceae & Nara & Grass \\
\hline Bambusa anundinacea Willd. & Poaceae & Magar & Grass \\
\hline Chrysopogon fulvus Choiv. & Poaceae & Dhaulu & Grass \\
\hline Cymbopogon citratus (DC.) Stapf. & Poaceae & Lemon grass & Grass \\
\hline Cymbopogon martini (Roxb.) Wats. & Poaceae & Dhub Khabbal, Talla & Grass \\
\hline Cynodon dactylon Pers. & Poaceae & Khabal, Dhub, Hariali & Grass \\
\hline Dactyloctenum aegyptium (Linn.) P. Beauv. & Poaceae & Madhana & Grass \\
\hline Dendrocalamus strictus Bl. & Poaceae & Baans & Grass \\
\hline Dicanthium annulatum Stapf. & Poaceae & Panni, Ganni & Grass \\
\hline Digitaria abludens (Roth. \& Schult) Veldk. & Poaceae & Bahia grass & Grass \\
\hline Digitaria bifasciculata auct. non Henr. & Poaceae & Love grass & Grass \\
\hline Digitaria ciliaris Koel & Poaceae & Kewari, Sheri & Grass \\
\hline Digitaria longiflora (Retz.) Pers. & Poaceae & Kanka-jariya & Grass \\
\hline Digitaria stricta Roth. ex Roem. \& Schult. & Poaceae & Bent grass & Grass \\
\hline Erianthus munja (Roxb.) Jeswiet & Poaceae & Kana, Munj & Grass \\
\hline Eriophorum comosum Wall. & Poaceae & Ghorbaggar & Grass \\
\hline Eulaliopsis binata Hubbard & Poaceae & Baggar & Grass \\
\hline
\end{tabular}




\begin{tabular}{|c|c|c|c|}
\hline Heteropogon contortus (L.) P. Beauv. & Poaceae & Lambu, Sariala, Kans & Grass \\
\hline Oplismenus bumannii (Retz.) Beauv. & Poaceae & Frass & Grass \\
\hline Oplismenus compositus (Linn.)P. Beauv. & Poaceae & Grass & Grundi \\
\hline Poa annua Linn. & Poaceae & Grass \\
\hline Saccharum benghalensis Retz. & Poaceae & Kahi, Kandali & Grass \\
\hline Sacchanum spontaneum Linn. & Poaceae & Fox tail grass & Grass \\
\hline Setaria glauca (Linn.) P. Beauv. & Poaceae & Italy grass & Grass \\
\hline Setaria italica (Linn.) P. Beauv. & Poaceae & Varuni & Grass \\
\hline Setaria verticillata (Linn.) P. Beauv. & Poaceae & Lunji \\
\hline Themeda anathera Hack. & Poaceae & Grass \\
\hline
\end{tabular}

Poaceae was the most dominant family with 30 species and 21 genera. Other main contributing families were Papilionaceae (10 species and 10 genera), Caesalpin iaceae (8 species and 3 genera), Euphorbeaceae (8 species and 7 genera), Apocynaceae (7 species and 5 genera), Acanthaceae (6 species and 4 genera) and Mimosaceae (6 species and 3 genera). Twenty-five families (23 dicots and 2 monocots) had only one species each (Table 2).

Sharma [9] recorded 1879 species of angiosperms from Punjab whereas Sharma et al. [10] recorded 526 species and Manhas et al. [11] recorded 202 species of angiosperms in their studies on Shiwaliks of Punjab. The dominance of plants from Poaceae, Euphorbiaceae and Apocynaceae families in the study site supports the harsh environmental conditions especially the water stress, because these plants have made morphological, anatomical and physiological modifications to overcome the drought conditions [12]. Dominance of Papilionaceae shows that these areas are nutrient deficient especially nitrogen $[11,12]$.

Table 2. List of important families of the study area

\begin{tabular}{|c|c|c|}
\hline Family & Species & Genera \\
\hline \multicolumn{3}{|l|}{ Dicotyledons } \\
\hline Papilionaceae & 10 & 10 \\
\hline Caesalpiniaceae & 8 & 3 \\
\hline Euphorbiaceae & 8 & 7 \\
\hline Apocynaceae & 7 & 5 \\
\hline Acanthaceae & 6 & 4 \\
\hline Mimosaceae & 6 & 3 \\
\hline Combret aceae & 5 & 2 \\
\hline Malvaceae & 5 & 2 \\
\hline Verbenaceae & 5 & 4 \\
\hline Amarantaceae & 4 & 2 \\
\hline Asteraceae & 4 & 4 \\
\hline Meliaceae & 4 & 4 \\
\hline Moraceae & 4 & 1 \\
\hline Rutaceae & 4 & 3 \\
\hline $\begin{array}{l}\text { Sapindaceae } \\
\end{array}$ & 4 & 2 \\
\hline Capparidaceae & 3 & 2 \\
\hline Lamiaceae & 3 & 3 \\
\hline Rhamnaceae & 3 & 2 \\
\hline Rubiaceae & 3 & 3 \\
\hline Urticaceae & 3 & 3 \\
\hline Anacardiaceae & 2 & 2 \\
\hline Boraginaceae & 2 & 2 \\
\hline Celastraceae & 2 & 2 \\
\hline Ebenaceae & 2 & 1 \\
\hline Menispermaceae & 2 & 2 \\
\hline Myrtaceae & 2 & 2 \\
\hline Oxalidaceae & 2 & 1 \\
\hline Tiliaceae & 2 & 2 \\
\hline Other (25) & 1 & 1 \\
\hline \multicolumn{3}{|l|}{ Monocotyledons } \\
\hline $\begin{array}{l}\text { Poaceae } \\
\end{array}$ & 30 & 21 \\
\hline Dioscoreaceae & 2 & 1 \\
\hline Liliaceae & 2 & 1 \\
\hline Palmae & 2 & 2 \\
\hline Commelinaceae & 1 & 1 \\
\hline Cyperaceae & 1 & 1 \\
\hline \multicolumn{3}{|l|}{ Gymnosperms } \\
\hline Pinaceae & 1 & 1 \\
\hline
\end{tabular}

The diversity of life forms was higher in the present study site (Figure 2). The most dominant life form was trees (36.9\%) followed by shrubs (22.7\%), grasses (17.1\%), herbs (13.6\%), climber (8.5\%) and sedges (1.1\%).

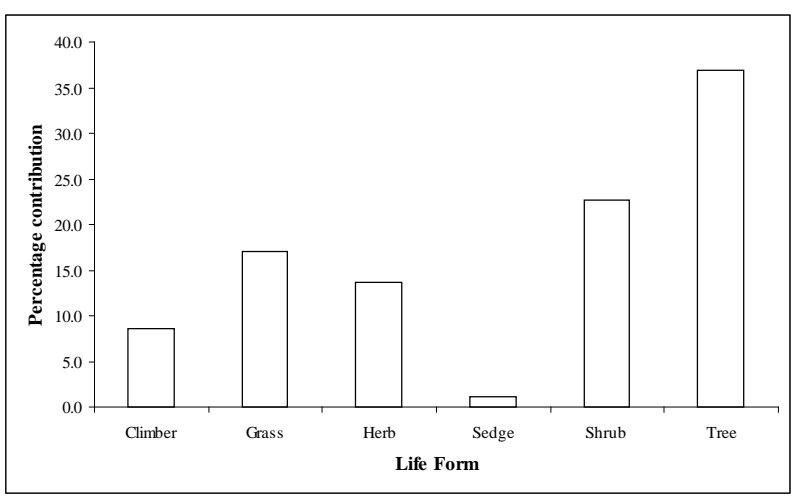

Figure 2. Contribution of various life forms to the flora of Hoshiarpur, Punjab

Generally, the high diversity of trees is associated with undisturbed tropical forests. But, forest sites in Punjab are highly degraded, which is also supported by the high shrub richness and dominance of grasses. Both these life forms prefer open forests with wide canopy gaps. High tree diversity of the present study may be ascribed to some of the protected areas (farm houses) of Hoshiarpur.

The vegetation of Kandi region of Punjab is very precious for the economy of the locals [13]. It provides fodder, fuelwood, non-wood forest products, medicinal plants etc. But, to use these natural resources in a sustainable way should be the main priority of the State Government and State Forest Department. Plantation programs and protection of vegetation from fire and grazing in different parts of Kandi belt are some of the initiatives taken by the forest department. In some parts of Kandi region joint forest management (JFM) has also been started with the help of locals.

Kandi region is a hilly terrain and major portion of the rainfall flows down without any underground water recharge due to lack of vegetation, as a result of this the entire Kandi region experiences drought like conditions in summer [14]. Various programs launched by the Punjab State Government and Department of Soil and Water Conservation, Punjab to build small check dams to collect water as well as slow down its flow during rainy season will certain ly help the local vegetation. Water harvesting may be the other option of recharging the ground water [15].

\section{Conclusion}

The present study deals with the floristic diversity of Kandi region of Hoshiarpur, Punjab. The plants were collected and then pressed in old newspapers/blotters. The 
pressing of plant specimens was carried out. The dried specimens were mounted on the herbarium sheets. Herbarium sheets were protected against damage from insects by poisoning them with $1 \%$ mercuric chloride. Naphthalene balls were also used to keep the insects away from the Herbarium. The floristic listing of species is very important for researchers and forest managers, as it tells us about the available resources of a region. This information can be utilized in future planning of sustainable utilization of these resources. Time to time assessment of species diversity also helps in studying the impact of temporal changes vis a vis change in land-use pattern, climate change etc.

\section{References}

[1] Shardha, Y. P. and Bagchi, J., An approach to meet the challenge of unpredictable hydrology in small catchments with reference to Kandi watershed area, Punjab, in National Symposium: Role of Earth Sciences in Integrated Development and Related Societal Issues, 2-4 November 2001, Lucknow. Special Publication No. 65: Vol. II. Kolkata. Geological Survey of India, 2001, 242 p.

[2] Champion, H.G. and Seth, S.K., A revised classification of the Forest Types in India. Manager Publications, GOI, New Delhi, 1968, 105-15.

[3] Stewart, J.L., Punjab Plants Comprising Botanical and Vernacular Name and Uses. 1869.

[4] Atchinson, J.E.T., A Catalogue of the Plants of the Punjab and Sindh. London, 1868.
[5] Bamber, C.J., Plants of the Punjab. A Descriiptive Key of the Flora of the Punjab, North-West Frontier Province and Kashmir. London, 1916.

[6] Parker, R.N., Forest Flora for the Punjab and Hazara and Delhi. London, 1918.

[7] Nair, N.C., Plants of record for the Punjab Plain. Bull. Bot. Sur. India, 21(1). 1-450. 1978.

[8] Sharma, M.L. and Khosla, P.K., The Grasses of Punjab and Chandigarh. Punjab University, Chandigarh, 1989, 1-296.

[9] Sharma M., Punjab Plants-Check List. Bishen Singh Mahinder Pal Singh, Dehradun, India, 1990.

[10] Sharma, M.L., Jerath, N. and Chadha, J., Angiosperms Biodiversity in the Shiwalik Ecosystem of Punjab. Punjab Science and Technology Department, Chandigarh, 256-450.

[11] Manhas, R.K., Singh, L. Vasistha, H.B. and Negi, M., Floristic Diversity of Protected Ecosystems of Kandi Region of Punjab, India. New York Science Journal, 3 (4). 96-103. 2010.

[12] Vasistha, H.B., Manhas, R.K., Singh, L., Negi, M. and Sharma, J. Impact of Disturbances on Biodiversity Status, Resource Availability and their Management for Sustainable Development in Kandi Area of Punjab. Punjab Forest Department, Chandigarh.

[13] Sarlach, R.S., Bhushan, B., Sharma, S.C., and Aulakh, P.S., Scope of raising aromatic, medicinal and spice crops in Kandi area of Punjab. Intemational Journal of Forest Usufructs Management, 1(7). 49-53. Jul. 2006

[14] Sheoran, P., Singh, S., Sidhu, B.S., Sardana, V., and Aulakh, P.S., Analysis of drought, dry and wet spells in Kandi region of Punjab. Journal of Research, 43(1). 14-16. Jan. 2006.

[15] Rana, D.S., Bawa, S.S., Singh, S. and Aulakh, P.S., Water harvesting, available technique for the rain-fed agro-ecosystem in foothill Shiwaliks of Punjab. Proceeding on "Efficient Water management for eco-friendly, sustainable and profitable agriculture" held at IARI, New Delhi, 2005, 153-154. 